\title{
DISPERSIVE ORDERING RESULTS
}

\author{
JAMES LYNCH, ${ }^{*}$ \\ GILLIAN MIMMACK** AND \\ FRANK PROSCHAN, ${ }^{* *}$ Florida State University
}

\begin{abstract}
A distribution $F$ is less dispersed than a distribution $G$ if $F^{-1}(\beta)-F^{-1}(\alpha) \leqq G^{-1}(\beta)-G^{-1}(\alpha)$ for all $0<\alpha<\beta<1 \quad(F \stackrel{\text { disp }}{\leftrightarrows} G)$.

We generalize a characterization of dispersive ordering of Shaked (1982) concerning sign changes of $F_{c}-G$, where $F_{c}$ is a translate of $F$. We then use this generalization plus total positivity to develop a simple proof of a characterization of dispersive distributions due to Lewis and Thompson (1981); a distribution $H$ is dispersive if $F \stackrel{\text { disp }}{\leqq} G \Rightarrow H * F \stackrel{\text { disp }}{\leqq} H * G$.
\end{abstract}

\section{Introduction}

Dispersive ordering is a partial ordering of distributions according to their degree of dispersion. In this note we (1) generalize a characterization of dispersive ordering by Shaked (1982) from the smooth case in which the distributions are absolutely continuous with interval supports, to the general case and (2) using this generalization, develop a simple proof of a characterization by Lewis and Thompson (1981) of distributions which preserve dispersive ordering under convolution.

More precisely, for a distribution function $F$, define $F^{-1}(\alpha)=$ $\inf \{t: F(t) \geqq \alpha\}(=\sup \{t: F(t)<\alpha\})$ for $0<\alpha<1$. Then we have the following.

Definition. A distribution function $F$ is less dispersed than is a distribution function $G$ if $F^{-1}(\beta)-F^{-1}(\alpha) \leqq G^{-1}(\beta)-G^{-1}(\alpha)$ for all $0<\alpha<\beta<1$; we write $F \stackrel{\text { disp }}{\leqq} G$.

\section{Extension of the Shaked characterization}

Let $S\left(x_{1}, \cdots, x_{n}\right)$ denote the number of sign changes of the sequence $x_{1}, \cdots, x_{n}$, where zero terms are discarded. Let $S(f)$ denote the number of sign changes of the

Received 2 March 1983; revision received 17 August 1983.

* Permanent address: Department of Statistics, Pennsylvania State University, University Park, PA 16802, U.S.A.

Research supported under Grant DAAG 29-82-K-0168 with the US Army Research Office.

$\dagger$ Postal address: Department of Statistics and Statistical Consulting Center, The Florida State University, Tallahassee, FL 32306, U.S.A.

Research supported under Contract AFOSR-82-K-0007 with the Air Force Office of Scientific Research, AFSC, USAF. 
function $f$ on $(-\infty, \infty)$; specifically,

$$
S(f)=\sup S\left[f\left(t_{1}\right), \cdots, f\left(t_{m}\right)\right],
$$

the supremum being taken over all $t_{1}<t_{2}<\cdots<t_{m}, m=2,3, \cdots$. Finally, let $S_{c} \equiv$ $S\left(F_{c}-G\right)$ for distribution functions $F$ and $G$.

Theorem 1. $F \stackrel{\text { disp }}{\leqq} G \Leftrightarrow$ for each real $c$, (a) $S\left(F_{c}-G\right) \leqq 1$ and (b) if $S_{c}=1$, then $F_{c}-G$ changes sign from - to + .

Proof. $\Rightarrow$ For fixed $c$, let $t_{0}$ satisfy $G\left(t_{0}\right)>F_{c}\left(t_{0}\right)$. Let $t_{*}=\inf \left\{t: G(s) \geqq F_{c}(s)\right.$ for $\left.t \leqq s \leqq t_{0}\right\}$. We shall show that $t_{*}=-\infty$. From this it will follow that $F_{c}-G$ can never change from + to - . This will complete the proof.

Suppose $t_{*}>-\infty$. By the definition of $t_{*}$ and the right continuity of $F$ and $G$, $G\left(t_{*}\right) \geqq F_{\mathrm{c}}\left(t_{*}\right)$ and $G\left(t_{*}-\varepsilon\right)<F_{\mathrm{c}}\left(t_{*}-\varepsilon\right)$ for sufficiently small $\varepsilon>0$. Fix such an $\varepsilon$; then let $\alpha=F_{c}\left(t_{*}-\varepsilon\right)$ and $\beta=G\left(t_{0}\right)$. It follows that

$$
G^{-1}(\alpha) \geqq t_{*}-\varepsilon \geqq F_{c}^{-1}(\alpha)
$$

and

$$
G^{-1}(\beta) \leqq t_{0}<F_{c}^{-1}(\beta)
$$

Thus

$$
G^{-1}(\beta)-G^{-1}(\alpha)<F_{c}^{-1}(\beta)-F_{c}^{-1}(\alpha),
$$

contradicting $F \stackrel{\text { disp }}{\leqq} G$. This proves the $\Rightarrow$ part.

$\Leftarrow$ Let $H_{n}$ denote the exponential distribution function with mean $\mu_{n} \rightarrow 0$ as $n \rightarrow \infty$. Let $F_{n}=H_{n} * F$ and $G_{n}=H_{n} * G$, where $*$ denotes convolution. By the variation diminishing theorem of Karlin (1968), the sign change properties of $F_{c}-G$ are inherited by $F_{n c}-G_{n}$. Thus $F_{n} \stackrel{\text { disp }}{\leqq} G_{n}$ by Theorem 2.1 of Shaked (1982) since $F_{n}$ and $G_{n}$ are both absolutely continuous with interval supports. Since $F_{n} \rightarrow F\left(G_{n} \rightarrow G\right)$ in distribution as $n \rightarrow \infty$, it follows that $F \stackrel{\text { disp }}{\leqq} G$.

\section{Dispersive distributions}

Definition. A distribution function $H$ is said to be dispersive if $H * F \stackrel{\text { disp }}{\leqq} H * G$ whenever $F \stackrel{\text { disp }}{\leqq} G$.

Next we present a simpler proof of Theorem 8 of Lewis and Thompson (1981) characterizing dispersive distribution functions. The simplification derives from the use of total positivity which is usually the appropriate way to treat problems of sign change.

Theorem 2. Let $H$ be a non-degenerate distribution function. Then $H$ is dispersive $\Leftrightarrow$ $H$ is absolutely continuous with a log concave density.

Proof. $\Leftarrow$ Let $F \stackrel{\text { disp }}{\leqq} G$. From Theorem 1 , for each real $c, S_{c}=S\left(F_{c}-G\right) \leqq 1$, with ato + sign change if $S_{c}=1$. Since $H$ has a log concave density, then by the variation diminishing theorem of Karlin (1968), Chapter 5, $S\left[(H * F)_{c}-(H * G)\right] \leqq 1$, with a - to + sign change if $S\left[(H * F)_{c}-(H * G)\right]=1$. It follows from Theorem 1 that $H * F \stackrel{\text { disp }}{\leqq}$ $H * G$

$\Rightarrow$ We use essentially Lewis and Thompson's argument, but avoid unnecessary details of their proof. Our proof is based on their elementary proof of the 'only if' part of their Theorem 7; we restate this for completeness. 
Lemma. Let $F$ be dispersive and twice continuously differentiable. Then $F$ has a $\log$ concave density.

To complete the proof of Theorem 2, let $H$ be dispersive and let $\Phi_{\sigma}$ denote a normal distribution with mean 0 and variance $\sigma^{2}$. From the first part of Theorem 2, note that $\Phi_{\sigma}$ is dispersive, and thus, $\Phi_{\sigma} * H$ is also dispersive. Since $\Phi_{\sigma} * H$ is infinitely differentiable, it follows from the lemma above that $\Phi_{\sigma} * H$ has a $\log$ concave density. Since $\Phi_{\sigma} * H \rightarrow H$ in distribution as $\sigma \rightarrow 0, H$ has a log concave density. (See Ibragimov (1956); note that a distribution is 'strongly unimodal' in Ibragimov's terminology iff its density is log concave.)

\section{References}

Ibragimov, I. A. (1956). On the composition of unimodal distributions. Theory Prob. Appl. 1, 255-260.

Karlin, S. (1968). Total Positivity, Vol. 1. Stanford University Press.

LEWIS, T. AND THOMPSON, J. W. (1981). Dispersive distributions, and the connection between dispersivity and strong unimodality. J. Appl. Prob. 18, 76-90.

SHAKED, M. (1982). Dispersive ordering of distributions. J. Appl. Prob. 19, 310-320. 